\title{
Solitary fibrous tumor of the liver mimicking malignancy
}

Hee Chul Nam ${ }^{1,2}$, Pil Soo Sung ${ }^{1,2}$, Eun Sun Jung ${ }^{3}$, and Seung Kew Yoon ${ }^{1,2}$

\begin{abstract}
${ }^{1}$ Department of Internal Medicine, ${ }^{2}$ The Catholic Liver Research Center, ${ }^{3}$ Department of Hospital Pathology, Seoul St. Mary's Hospital, College of Medicine, The Catholic University of Korea, Seoul, Korea
\end{abstract}

A 45-year-old male patient presented to our clinic for an intrahepatic mass observed on ultrasonography. His blood test, including liver function test, results were within normal ranges. Viral hepatitis markers, alpha-fetoprotein, des-gamma-carboxy prothrombin, and cancer antigen 19-9 levels were all unremarkable.

Magnetic resonance imaging (MRI) with Primovist (Bayer Healthcare, Berlin, Germany) enhancement revealed a $2.8 \mathrm{~cm}$ tumor (Fig. 1). The mass was well enhanced in the arterial phase, became isointense during the portal venous phase, and was identified as a defect in the hepatobiliary phase. The tumor's radiologic features were difficult to differentiate from that of a malignant tumor such as hepatocellular carcinoma (HCC). However, the patient did not have any risk factors for the development of HCC, and serum tumor markers were within normal ranges. The histological results of a fine-needle liver biopsy for diagnosis showed stromal fibrosis and spindle-shaped tumor with high cellularity (Fig. 2). Immunohistochemical analysis revealed strong positivity for $\mathrm{CD}_{34}$ and $\mathrm{CD}_{99}$, but not for
Received: December 10, 2018

Revised : January 13, 2019

Accepted: January 13, 2019

\section{Correspondence to}

Seung Kew Yoon, M.D.

Tel: +82-2-2258-2073

Fax: +82-2-3481-4025

E-mail:yoonsk@catholic.ac.kr
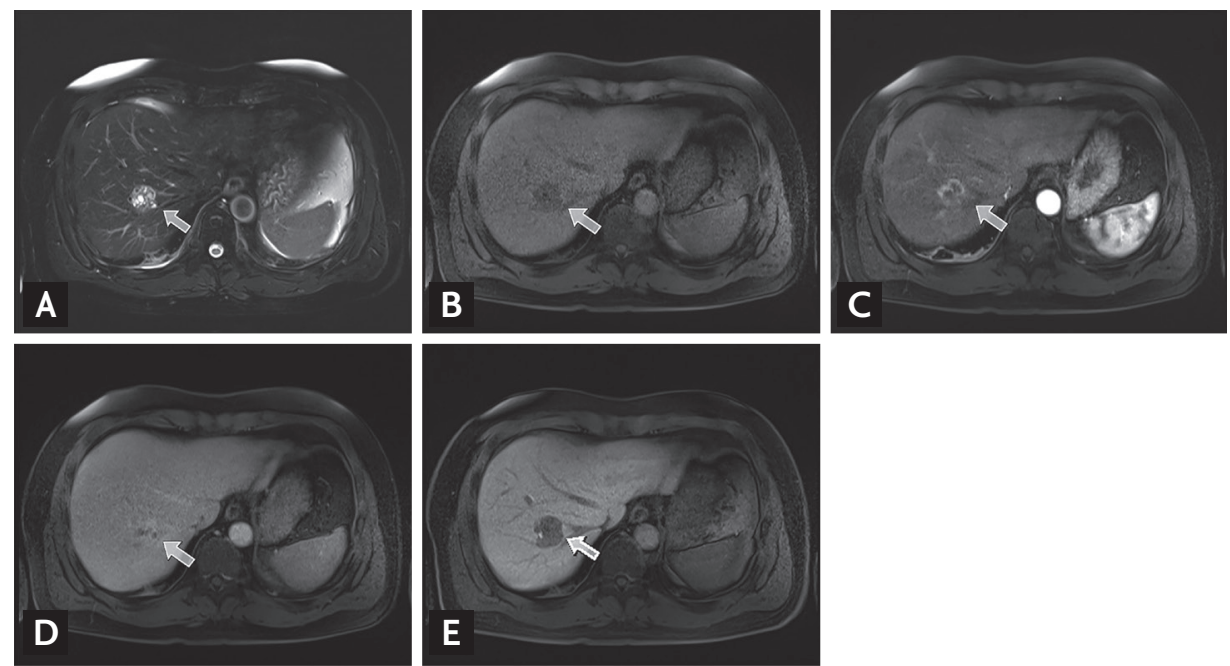

Figure 1. A magnetic resonance imaging scan revealed a mass at S8. (A) Multiple cystic portions within the tumor presented as dots with high signal intensities in the T2-weighted image. (B) T1-weighted image showing the hypoechoic mass. (C) After Primovist enhancement, the mass was well-enhanced in the arterial phase and (D) became isointense to the surrounding parenchyme during the portal venous phase. (E) The tumor appeared as a defect during the hepatobiliary phase. All arrows indicated the intrahepatic mass. 

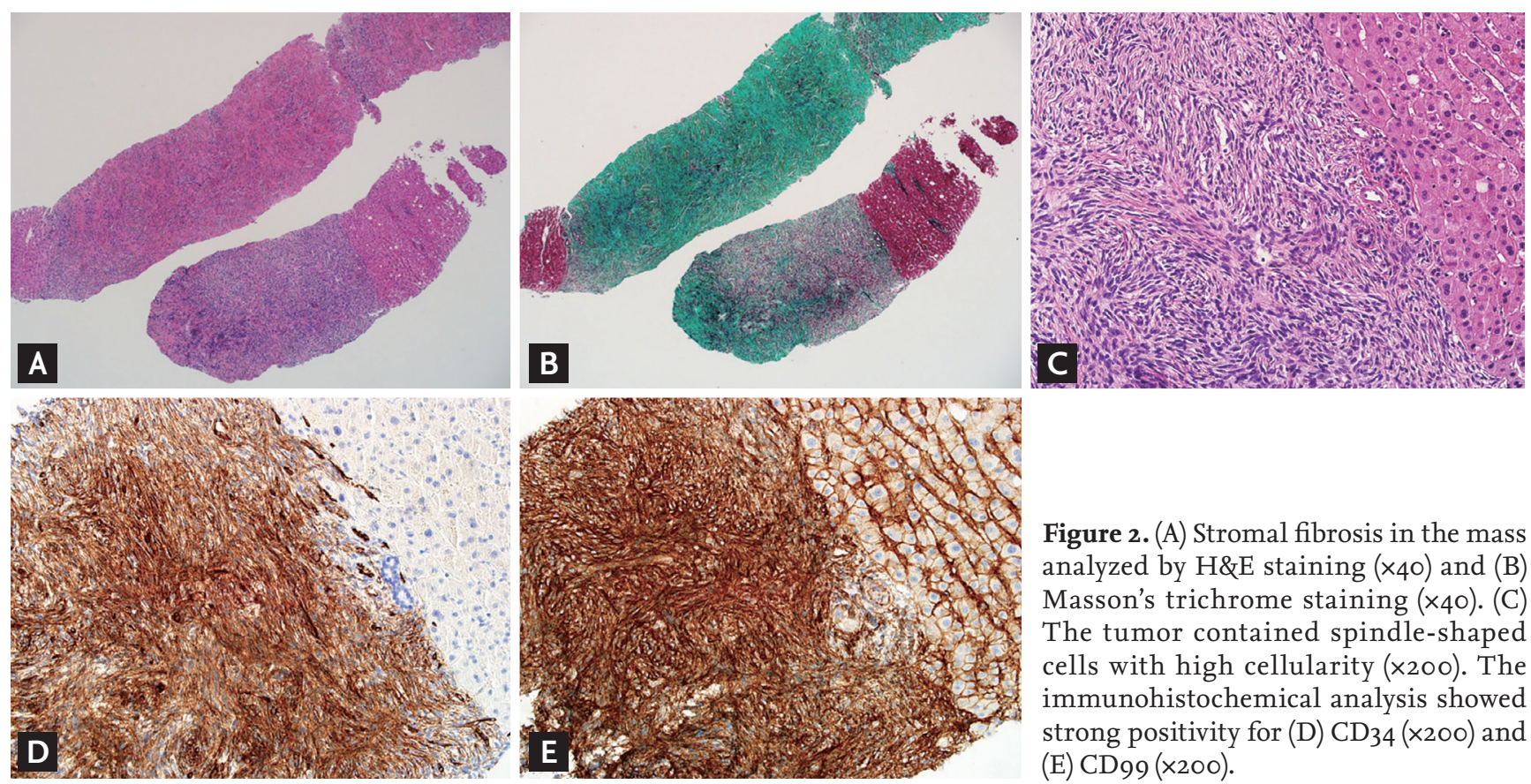

Figure 2. (A) Stromal fibrosis in the mass analyzed by $\mathrm{H} \& \mathrm{E}$ staining $(\times 4 \mathrm{O})$ and $(\mathrm{B})$ Masson's trichrome staining $(\times 40)$. (C) The tumor contained spindle-shaped cells with high cellularity $(\times 200)$. The immunohistochemical analysis showed strong positivity for (D) CD34 (×200) and (E) CD99 (×200).

CD117, actin, or Ki-67. The histological results including an immunohistochemistry stain revealed typical microscopic features of solitary fibrous tumor (SFT). Because the tumor size was small, and there was no evidence of mitosis or nuclear pleomorphism, we decided on a close follow-up with ultrasonography without intervention.

SFT is a rare neoplasm arising from the mesenchymal cells. The liver is one of the most uncommon sites for SFT development and reported cases of SFT in the liver have been limited. Most patients with SFTs are asymptomatic and present with a benign clinical course. However, careful monitoring is needed because malignant change can occur.

Here, we describe a very rare intrahepatic benign tumor that mimicked malignancy. Because MRI scans were not sufficient to differentiate between a malignant and benign tumor in this case, an accurate diagnosis was obtained through a biopsy.

We received informed consent from the patient for the publication of this case.

\section{Conflict of interest}

No potential conflict of interest relevant to this article was reported. 